
\title{
25 Research Square \\ Distinct Infection Forms of SARS-CoV-2 Among People Living With HIV
}

\section{Mengmeng Wu}

Zhongnan Hospital of Wuhan University

\section{Fangzhao Ming}

Wuchang District Center for Disease Control and Prevention

\section{Songjie Wu}

Zhongnan Hospital of Wuhan University

\section{Yanbin Liu}

Zhongnan Hospital of Wuhan University

\section{Xiaoxia Zhang}

Zhongnan Hospital of Wuhan University

\section{Wei Guo}

Zhongnan Hospital of Wuhan University

\section{Gifty Marly}

Nanjing Medical University

Weiming Tang ( $\square$ Weiming_tang@med.unc.edu )

Southern Medical University

\section{Ke Liang}

Zhongnan Hospital of Wuhan University

\section{Research Article}

Keywords: SARS-CoV-2, People living with human immunodeficiency virus (PLWH), asymptomatic carrier, unapparent infector

Posted Date: July 19th, 2021

DOI: https://doi.org/10.21203/rs.3.rs-569883/v2

License: (c) (i) This work is licensed under a Creative Commons Attribution 4.0 International License. Read Full License 


\section{Abstract \\ Background}

People living with HIV (PLWH) are immunodeficient, it is vague if they are more susceptible to SARS-CoV2 infection than HIV negative individuals.

\section{Methods}

In this cross-sectional study, 857 PLWH and 1048 HIV negative individuals were enrolled from the Wuchang district in Wuhan, China. We compared the total rate of SARS-CoV-2 infection, the rate of COVID-19, asymptomatic carriers, and unapparent infectors in the two groups. The risk factors associated with SARS-CoV-2 infection among PLWH were explored.

\section{Results}

Fourteen out of 857 (1.63\%) PLWH were infected with SARS-CoV-2, while 68 of 1048 (6.49\%) HIV negative individuals were infected. In PLWH, there were 6 confirmed COVID-19 (0.70\%), 4 asymptomatic carriers $(0.47 \%)$ and 4 unapparent infectors $(0.47 \%)$. In the HIV negative group, the cases of COVID- 19 , asymptomatic carrier, and unapparent infector were $5(0.48 \%), 0(0.00 \%)$, and $63(6.01 \%)$, respectively. After adjusting for age, gender, and chronic comorbidities, the rate of SARS-CoV-2 infection in PLWH was lower than that in HIV negative group $(1.96 \%$ vs $5.74 \%, P=0.001)$. The morbidity of COVID-19 was similar between the two groups $(P=0.107)$, but the rate of unapparent infection in PLWH was lower than that in the HIV negative group $(0.54 \%$ vs $5.46 \%, P=0.001)$. Older age $(\mathrm{aOR}=4.50,95 \% \mathrm{Cl}: 1.34-15.13, \mathrm{P}=0.015)$ and Ols $(\mathrm{aOR}=9.59,95 \% \mathrm{Cl}: 1.54-59.92, \mathrm{P}=0.016)$ were risk factors for SARS-CoV-2 infection among PLWH.

\section{Conclusions}

PLWH has different infection forms of SARS-CoV-2 compared with the general population. Older age and Ols were considered to driving causes of SARS-CoV-2 infection among PLWH.

\section{Background}

By May 21st, 2021, a total of 165,580,045 confirmed cases and 3,431,857 deaths had been reported globally since the coronavirus disease 2019 (COVID-19) outbreak.[1] Due to the immune deficiency caused by the human immunodeficiency virus (HIV), people living with HIV (PLWH) were thought to be more vulnerable to the severe acute respiratory syndrome coronavirus 2 (SARS-CoV-2).[2] However, the current evidence is still lacking and inconsistent, as some studies have indicated that the morbidity of COVID-19 in PLWH is not higher than in the general population.[3] In addition, most of the previous 
studies did not take asymptomatic carriers and unapparent infectors into consideration, and they are largely missed in the existing literature.[4, 5] Whether the evidence will be similar to the existing literature after considering these two groups of individuals are still vague.

This study aimed to further investigate the prevalence and associated risk factors of SARS-CoV-2 infection in PLWH and HIV negative individuals in Wuhan, China, the earliest epicenter of COVID-19.

\section{Methods}

\section{Study design and participants recruitment}

As an extension of our former work[6, 7], this cross-sectional study has consisted of two groups of the population that participated in the seroepidemiological survey of SARS-CoV-2 in Wuchang district, Wuhan. The investigation was proceeded from May 1, 2020, to May 31, 2020. All the participants (age $\geq$ 18 years old) were lived in the Wuchang district for at least 1 month from December 1, 2019, to April 8, 2020.

All PLWH who were managed by the Wuchang district center for disease control and prevention (CDC) were recruited. The participants who were tested positive for HIV have been reported to Wuchang CDC through the China National HIV/AIDS Comprehensive Response Information Management System (CRIMS).

For the general group, a two-stage cluster sampling method was used to recruit the study population. Selecting communities as primary sampling units (PSUs) at the first stage and families at the second stage. Overall, all communities were certainty PSUs and 11 communities were selected with probability proportional to the size sampling method. Within each community, 36 households were selected by systematic random sampling method and all members of the households were invited to participate in the study. If individuals of a certain age group were missing, we swallowed the sample randomly to ensure that the age structure of the sample was similar to the natural structure of the population.

\section{Data collection}

For PLWH, demographic information includes age, gender, chronic comorbidities, the mode of HIV acquisition, antiretroviral (ARV) regimens, current opportunistic infections (Ols). ARV regimens were obtained from CRIMS. Basic information about HIV negative participants was collected through a questionnaire. All participants were inquired of COVID-19 history, and we double-checked the name and identification card number with that of recorded COVID-19 patients in the CDC information management systems. All SARS-CoV-2 infections are diagnosed according to the 8th edition of clinical practice guidelines for COVID-19 in China.[8] The total SARS-CoV-2 infection rate including the rate of COVID-19, asymptomatic carrier, and unapparent infector.

\section{Definitions}


Chronic comorbidities include hypertension, diabetes, chronic respiratory disease, cancer, and any other chronic disease that has been diagnosed. The definition of Ols was referring to the guideline formulated by the U.S. Department of Health and Human Services (DHHS).[9] The Asymptomatic carrier is defined as a patient who does not have clinical manifestations of COVID-19 but the nucleic acid is positive. The unapparent infector is defined as a patient who does not have clinical manifestations and nucleic acid negative but serum antibody for SARS-CoV-2 was positive.

\section{Laboratory procedures}

The CD4 + T lymphocyte count (CD4 count) and HIV viral load (HIV-VL) were detected for PLWH. All recruited general individuals received HIV antibody screening tests. Methods for laboratory confirmation of SARS-CoV-2 infection included: respiratory specimens SARS-CoV-2 real-time fluorescence Polymerase Chain Reaction (RT-PCR), serum SARS-CoV-2 IgM/lgG antibody colloidal gold test, and magnetic particle chemiluminescence (qualitative result). The detection kits were provided by Shengxiang Biotechnology Co., LTD, and Guangzhou Wanfu Biotech Co., LTD. The kits were approved by the China Food and Drug Administration (FDA). In this study, swab nucleic and serum antibodies ( $\mathrm{IgM} / \mathrm{lgG}$ ) were detected for all subjects. All positive specimens (nucleic acid, IgM, or IgG positive) were sent to China CDC for confirmation.

\section{Statistical analysis}

Continuous variables were expressed as means (SD) or median (interquartile range) and categorical variables were expressed as frequency and percent. Comparisons of continuous variables were assessed using the independent sample T-test or Wilcoxon rank-sum test, while categorical variables were assessed using the $\chi^{2}$ test or the Fisher exact test. We calculated the crude rate and $95 \%$ confidence interval ( $95 \%$ $\mathrm{CI}$ ) of SARS-CoV-2 infection to estimated using the exact binomial distribution. Then we used a logistic regression model to calculate the adjusted rate and $95 \% \mathrm{Cl}$ of SARS-CoV-2 infection after adjusting for age, gender, and chronic comorbidities to compare the difference in SARS-CoV-2 infection rate between PLWH and the HIV negative group in the Wuchang district. Univariate and multivariable modified Poisson regression methods were used to explore the risk factors associated with PLWH co-infected with SARSCoV-2.

Statistical significance was defined as a two-sided p-value of less than 0.05. All analyses were conducted using STATA version 13.0 (STATA Corporation, College Station, Texas) and IBM SPSS Statistics (Version 26.0) software.

\section{Results}

\section{Participants enrolled in the study}

Totally, 910 PLWH were managed in Wuchang CDC. But 2 were excluded because they were living abroad during the closure of Wuhan and 51 refused to participate in this study. The control group consisted of 1 , 
100 HIV-negative individuals of the general population selected from residents living in the Wuchang district, and 52 refused to participate in the study. In total, 857 PLWH and 1048 HIV negative participants were enrolled in this study. The PLWH were younger than HIV negative subjects $(P=0.001)$. The PLWH were predominantly male $(P=0.001)$ and had fewer comorbidities than the HIV negative population $(P=$ 0.001) (Table 1).

Table 1

SARS-CoV-2 infection between HIV positive and negative group in Wuchang District, 2020 $(\mathrm{N}=1905)$

\begin{tabular}{|llll|}
\hline & $\begin{array}{l}\text { HIV-positive group } \\
(\mathbf{n}=\mathbf{8 5 7 )}\end{array}$ & $\begin{array}{l}\text { HIV-negative group } \\
(\mathbf{n = 1 0 4 8 )}\end{array}$ & P-value \\
\hline Age, year (means \pm SD) & $39.7 \pm 14.1$ & $47.4 \pm 14.2$ & 0.001 \\
\hline Gender (\%) & & & 0.001 \\
\hline Male & $774(90.32)$ & $451(43.03)$ & \\
\hline Female & $83(9.68)$ & $597(56.97)$ & \\
\hline Chronic Comorbidities (\%) & $51(5.95)$ & $255(24.33)$ & 0.001 \\
\hline Total SARS-CoV-2 infection (\%) & $14(1.63)$ & $68(6.49)$ & 0.001 \\
\hline COVID-19 & $6(0.70)$ & $5(0.48)$ & 0.068 \\
\hline Asymptomatic Carriers & $4(0.47)$ & $0(0.00)$ & 0.040 \\
\hline Unapparent Infectors & $4(0.47)$ & $63(6.01)$ & 0.001 \\
\hline IgM (+) IgG (-) & $1(0.12)$ & $11(1.05)$ & \\
\hline IgM (-) IgG (+) & $2(0.23)$ & $29(2.77)$ & \\
\hline IgM (+) IgG (+) & $1(0.12)$ & $23(2.19)$ & \\
\hline
\end{tabular}

\section{SARS-CoV-2 infection between PLWH and HIV negative group}

In PLWH,there were 6 confirmed COVID-19 (0.70\%), 4 asymptomatic carriers $(0.47 \%)$ and 4 unapparent infectors $(0.47 \%)$. In HIV negative group, the number of confirmed COVID-19, asymptomatic carriers, and unapparent infectors were $5(0.48 \%), 0(0.00 \%)$, and $63(6.01 \%)$, respectively. The crude SARS-CoV-2 infection rate was 1.63\% (14/857) in PLWH and 6.49\%(68/1048)in HIV negative group. After adjusting for age, gender, and chronic comorbidities by the logistic regression model, the adjusted rate of SARS-CoV-2 infection in PLWH (1.96\%, 95\% Cl: 0.90-3.01) was lower than that in the HIV negative group (5.74\%, 95\%Cl: $4.31-7.17)(P=0.001)$. But the adjusted rate of COVID-19 was no difference between PLWH 
(1.10\%, 95\% Cl: $0.11-2.10)$ and HIV negative group $(0.37 \%, 95 \% \mathrm{Cl}: 0.04-0.69)(P=0.107)$. The adjusted rate of unapparent infection in PLWH $(0.54 \%, 95 \% \mathrm{Cl}: 0.00-1.07)$ were also lower than in HIV negative group (5.46\%, 95\%Cl: 4.02-6.91) ( $\mathrm{P}=0.001)$ (Table 2).

Table 2खComparison of SARS-CoV-2 infection between PLWH and HIV negative group in Wuchang District $(\mathrm{N}=1905)$

\begin{tabular}{|llll|}
\hline & PLWH & HIV negative group $(\mathrm{n}=1048)$ & P value \\
& $(\mathrm{n}=857)$ & & \\
Total SARS-CoV-2 infection & & & \\
Crude rate $(\%, 95 \% \mathrm{Cl}){ }^{\#}$ & $1.63(0.78-2.48)$ & $6.49(4.99-7.98)$ & 0.001 \\
Adjusted rate $(\%, 95 \% \mathrm{Cl})$ * & $1.96(0.90-3.01)$ & $5.74(4.31-7.17)$ & \\
COVID-19 & & & 0.107 \\
Crude rate $(\%, 95 \% \mathrm{Cl}){ }^{\#}$ & $0.70(0.14-1.26)$ & $0.48(0.06-0.90)$ & \\
Adjusted rate $(\%, 95 \% \mathrm{Cl})$ * & $1.10(0.11-2.10)$ & $0.37(0.04-0.69)$ & $\mathrm{NA}$ \\
Asymptomatic carriers & & & \\
Crude rate $(\%, 95 \% \mathrm{Cl}){ }^{\#}$ & $0.47(0-0.92)$ & 0 & 0.001 \\
Adjusted rate $(\%, 95 \% \mathrm{Cl})$ * & $\mathrm{NA}$ & $\mathrm{NA}$ & \\
Unapparent infectors & & $6.01(4.57-7.45)$ & \\
Crude rate $(\%, 95 \% \mathrm{Cl}){ }^{\#}$ & $0.47(0-0.92)$ & $5.46(4.02-6.91)$ & \\
Adjusted rate $(\%, 95 \% \mathrm{Cl})$ * & $0.54(0.00-1.07)$ & & \\
\hline
\end{tabular}

\#Confidence intervals estimated using exact binomial distribution. ${ }^{*}$ The adjusted rate was obtained after adjusting for age, gender, and chronic comorbidities using logistic regression.

\section{Comparison of the characteristics of SARS-CoV-2 infected and non-infected PLWH}

PLWH infected with SARS-CoV-2 had older age than those who did not infect with SARS-CoV-2 (53.5 years vs 35.0 years, $P=0.005)$, and a higher rate of chronic comorbidities $(P=0.048)$. In addition, PLWH with Ols had a higher SARS-CoV-2 infection rate (14.29\%) compared to PLWH without Ols $(0.59 \%)(P=$ 0.005). The two groups were similar in ARV regimens, gender, mode of HIV acquisition, CD4 count, and HIV-VL (Table 3). 
Table 3

Demographic features of enrolled PLWH in Wuhan, China, 2020 ( $N=857)$

\begin{tabular}{|c|c|c|c|}
\hline Characteristics & $\begin{array}{l}\text { Uninfected SARS-CoV-2 } \\
(n=843)\end{array}$ & $\begin{array}{l}\text { Infected SARS-CoV-2 } \\
(n=14)\end{array}$ & P-value \\
\hline Age (median interquartile range) & $35.0(29.00-49.00)$ & $53.5(42.25-61.00)$ & 0.005 \\
\hline Gender (\%) & & & 1.000 \\
\hline Male & $761(90.27)$ & $13(92.86)$ & \\
\hline Female & $82(9.73)$ & $1(7.14)$ & \\
\hline Chronic Comorbidities (\%) & $49(5.81)$ & $3(21.43)$ & 0.048 \\
\hline \multicolumn{4}{|l|}{ Mode of HIV acquisition (\%) } \\
\hline Heterosexual transmission & $196(23.25)$ & $3(21.43)$ & 1.000 \\
\hline Homosexual & $637(75.56)$ & $11(78.57)$ & 1.000 \\
\hline Other & $10(1.19)$ & $0(0.00)$ & 1.000 \\
\hline Ols (\%) & & & 0.005 \\
\hline Yes & $5(0.59)$ & $2(14.29)$ & \\
\hline No & $838(99.41)$ & $12(85.71)$ & \\
\hline CD4 count (cells $/ \mu \mathrm{L})$ & & & 0.074 \\
\hline$<100$ & $26(3.08 \%)$ & $2(14.29 \%)$ & \\
\hline$\geq 100$ & $817(96.92 \%)$ & $12(85.71 \%)$ & \\
\hline HIV-VL (copies/mL) & & & 1.000 \\
\hline$<20$ & $618(73.31 \%)$ & $10(71.43 \%)$ & \\
\hline$\geq 20$ & $225(26.69 \%)$ & $4(28.57 \%)$ & \\
\hline \multicolumn{4}{|l|}{ ARV regimens (\%) } \\
\hline NRTI + NNRTI & $699(82.92)$ & $13(92.86)$ & 0.485 \\
\hline Pls-based & $78(9.25)$ & $0(0.00)$ & 0.629 \\
\hline INIs-based & $51(6.05)$ & $0(0.00)$ & 0.587 \\
\hline Not on ARV & 15 (1.78) & $1(7.14)$ & 1.000 \\
\hline
\end{tabular}




\section{Risk factors of SARS-CoV-2 infection among PLWH}

Univariate regression analysis suggested HIV and SARS-CoV-2 co-infection is associated with elder age (age $\geq 50, \mathrm{OR}=8.36,95 \% \mathrm{Cl}: 3.01-23.22, \mathrm{P}=0.001)$, chronic comorbidities $(\mathrm{OR}=4.70,95 \% \mathrm{Cl}: 1.42-15.58$, $\mathrm{P}=0.011)$ and $\mathrm{Ols}(\mathrm{OR}=23.05,95 \% \mathrm{Cl}: 5.93-89.57, \mathrm{P}=0.001)$, and $\mathrm{CD} 4$ count less than $100 / \mu \mathrm{L}(\mathrm{OR}=$ $0.19,95 \% \mathrm{Cl}: 0.05-0.76, \mathrm{P}=0.019)$. Multivariable regression analysis further suggested that age $(\mathrm{aOR}=$ $4.50,95 \% \mathrm{Cl}: 1.34-15.13, \mathrm{P}=0.015)$ and Ols $(\mathrm{aOR}=9.59,95 \% \mathrm{Cl}: 1.54-59.92, \mathrm{P}=0.016)$ were still associated with SARS-CoV-2 infection, after adjusted for gender, chronic comorbidities, mode of HIV acquisition, CD4 count, HIV-VL and ARV regimens (Table 4). 
Table 4

The risk factors of SARS-CoV-2 infection among PLWH, in Wuhan, China, 2020 ( $N=857)$

\begin{tabular}{|c|c|c|c|c|}
\hline \multirow[t]{2}{*}{ Characteristics } & \multicolumn{2}{|l|}{ Univariate analysis } & \multicolumn{2}{|l|}{ Multivariable analysis* } \\
\hline & OR $(95 \% \mathrm{Cl})$ & P-value & Adjusted OR (aOR) $(95 \% \mathrm{Cl})$ & P-value \\
\hline \multicolumn{5}{|l|}{ Age (years) } \\
\hline $18-49$ & 1.00 & & 1.00 & \\
\hline$\geq 50$ & $8.36(3.01-23.22)$ & 0.001 & $4.50(1.34-15.13)$ & 0.015 \\
\hline \multicolumn{5}{|l|}{ Gender } \\
\hline Male & 1.00 & & 1.00 & \\
\hline Female & $0.65(0.12-3.50)$ & 0.616 & $0.82(0.07-9.12)$ & 0.872 \\
\hline
\end{tabular}

\section{Chronic Comorbidities}

No

1.00

1.00

Yes

$4.70(1.42-15.58)$

0.011

$2.17(0.52-9.12)$

0.290

Mode of HIV acquisition

Non-MSM

1.00

1.00

MSM

$0.36(0.11-1.19)$

0.093

$0.53(0.13-2.11)$

0.617

Ols

No

1.00

1.00

Yes

23.05 (5.93-89.57)

0.001

$9.59(1.54-59.92)$

0.016

CD4 count (cells $/ \mu \mathrm{L}$ )

$<100$

1.00

1.00

$\geq 100$

$0.19(0.05-0.76)$

0.019

$0.27(0.04-1.96)$

0.197

HIV-VL (copies/mL)

$<20$

1.00

1.00

$\geq 20$

$1.38(0.46-4.17)$

0.567

$0.98(0.26-3.79)$

0.985

ARV regimens

Yes

1.00

1.00

No

0.66 (0.04-11.00)

0.769

$1.00(1.00-1.00)$

MSM: men who have sex with men. *Each association was mutually adjusted for the other characteristics in the table. 


\section{Discussion}

This study extended the existing literature by including all three forms of SARS-CoV-2 infection and investigated the risks of total SARS-CoV-2 infection among PLWH. In May 2020 (one month after the first SARS-CoV-2 pandemic was contained in China), a cross-sectional survey showed that the positive incidence of antibody for SARS-CoV-2 in Wuhan was 4.43\%[10], which is similar to the total SARS-CoV-2 infection incidence among HIV negative group in our study (6.49\%). And our study showed that the total SARS-CoV-2 infection rate was lower among PLWH (1.63\%) than HIV negative group, but PLWH has different infection forms of SARS-CoV-2 compared with the HIV negative group. The rate of COVID-19 reported in Wuhan was $0.45 \%[3]$ and was similar to that in both HIV negative group (0.48\%) and PLWH $(0.70 \%)$ that we surveyed. There was no difference in the rate of COVID-19 between the PLWH and HIV negative group in our study, which has been demonstrated in another study[11].

But we found that PLWH had more asymptomatic carriers than the control group. Although asymptomatic carriers were not found in the control group, the rate of asymptomatic carriers in PLWH $(0.46 \%)$ was still higher than reported previous studies from Wuhan among the general population (8/61437, $0.013 \%$ in Wuchang district) and $(221 / 158403,0.001 \%$ in Wuhan city).[12, 13] Two reasons may have led to this phenomenon. First, immune deficiency in PLWH causes the body to clear the SARSCoV-2 more slowly than in HIV negative patients.[14-16] In the general population, the median time for virus shedding in asymptomatic carriers was 19 days, and that in symptomatic infected persons was 14 days.[14] Among PLWH, the median time of virus shedding was 18 days in $68 \%$ of patients, but SARSCoV-2 was still detected in 32\% of patients 13-45 days after COVID-19 was diagnosed.[17] Secondly, PLWH may have weaker relevant clinical manifestation for their lower immunity when they were infected with SARS-CoV-2.[18, 19] This may have increased their chances of just being asymptomatic carriers at the initial stage of SARS-CoV-2 infection.

In our study, the unapparent infection of SARS-CoV-2 among PLWH (0.47\%) was less than that in the HIV negative group (6.01\%). After adjustment, there were still differences between the unapparent infection in the two groups. We considered two potential reasons that may have led to this phenomenon. $B$ cell dysfunction appears during HIV infection resulting in impaired antibody responses to vaccines.[20] The first reason is that lower immunity of PLWH leads to insufficient antibody production than found in HIV negative people. The other reason we considered is that serum levels of antibodies descended faster in PLWH than in members of the general population. One study in Chongqing province of China with similar observation showed that antibodies decreased by more than $70 \%$ after two months in $90 \%$ of SARS-CoV2 infectors, with a faster decrease rate especially in asymptomatic carriers compared to symptomatic persons in the general population.[14] Our former study also showed the positive conversion rate of IgG for SARS-CoV-2 was relatively lower and quickly lost in PLWH.[21] 
Our study showed PLWH who had Ols are more likely to be infected with SARS-CoV-2. As is known to all, PLWH with Ols has severely impaired immunity, which means PLWH is easier to get other infections.[22] Some studies suggested that PLWH with tuberculosis infection are more susceptible to SARS-CoV-2 infection. $[23,24]$ However, reports about PLWH with Ols and SARS-CoV-2 infection are limited.[25] On the other hand, the common Ols in PLWH are tuberculosis, pneumocystis pneumonia, and bacterial pneumonia, all of which can cause lung damage,[26] and injure the local pulmonary immunity. It is noteworthy that, lower local pulmonary immunity could enhance SARS-CoV-2 infection in the theory. In addition, our data suggested that a lower current CD4 count contributes to a higher risk of acquiring SARS-CoV-2 infection. Thought the difference between the PLWH with SARS-CoV-2 and PLWH without SARS-CoV-2 was of marginal statistical significance, but the univariate regression analysis of risk factors for SARS-CoV-2 infection demonstrated that CD 4 count $\otimes 100 / \mu \mathrm{L}$ was one of the risk factors.

At early outbreak, many scholars have speculated that ARV drugs have therapeutic and preventive effects on COVID-19.[27, 28] But a study in Spain found ARV drugs could not reduce the morbidity of COVID-19 among PLWH.[29] Also in a randomized controlled open-label trial suggested that no benefit was observed with lopinavir-ritonavir in COVID-19 patients.[30] However, these subjects have focused on the efficacy of ARV drugs in the treatment or prevention of COVID-19 and did not suggest if ARV drugs can reduce the incidence of SARS-CoV-2 infection. Our study suggested the ARV drugs do not provide prophylaxis for SARS-CoV-2 infection among PLWH.

Our study has several limitations. First, our investigation was a cross-sectional study and hence may not reflect the conditions at the early stage of the SARS-CoV-2 pandemic in Wuhan. Another limitation is that the number of enrolled PLWH is limited, and whether the two important indicators (HIV-VL and CD4 count) that assess the immune status of PLWH are related to HIV co-infection remains to be further discussed. Third, our study sample is relatively small, which limited us to conduct more significant analyses. In addition, due to no asymptomatic infection was detected in the HIV negative group, we could not calculate the adjusted rate and $95 \% \mathrm{Cl}$ of asymptomatic and compare the difference between the two groups by Logistic model. Finally, although the serological antibody test had a certain false-positive rate, we tested each positive specimen again.

\section{Conclusions}

PLWH were more likely to be asymptomatic and the seroprevalence of antibodies in PLWH is lower than in HIV positive population after infected SARS-CoV-2. Among PLWH, the elderly and those with Ols need to pay more attention to personal protection against SARS-CoV-2 infection. The ARV drugs do not provide prophylaxis for SARS-CoV-2 infection among PLWH.

\section{Abbreviations}

PLWH 
people living with HIV. SARS-CoV-2:severe acute respiratory syndrome coronavirus 2. COVID-19:corona virus disease 2019. HIV:human immunodeficiency virus. AIDS:acquired immune deficiency syndrome. aOR:after adjust rate. $95 \% \mathrm{Cl}: 95 \%$ confidence interval. CDC:disease control and prevention. CRIMS:China national HIV/AIDS comprehensive response information management system. PSUs:primary sampling units. ARV:antiretroviral. Ols:opportunistic infections. DHHS:health and human services. CD4 count:CD4 + T lymphocyte count. HIV-VL:HIV viral load. RT-PCR:real-time fluorescence polymerase chain reaction. IgM:immune globulin M. IgG:immune globulin G. FDA:food and drug administration. SD:standard deviation.

\section{Declarations}

\section{Ethics approval and consent to participate}

This study was approved by the Ethics Committee of Zhongnan Hospital affiliated with Wuhan University (2020062), and informed consent was obtained. All methods were performed in accordance with the $8^{\text {th }}$ edition of clinical practice guidelines for COVID-19 in China.

\section{Consent for publication}

Institutional consent.

\section{Availability of data and materials}

The datasets generated and analyzed during the current study are available from the corresponding author on reasonable request.

\section{Competing interests}

The authors declare that have no competing interests.

\section{Funding}

This work was supported by the National Key Research and Development Program of China (2017YFE0103800), the National Nature Science Foundation of China (81903371), NIMH (R34MH119963), the National Science and Technology Major Project (2018ZX10101-001-001-003), and Special Found on Prevention and Control of New Coronary Pneumonia in Guangdong Universities (2020KZDZX1047), Medical Science and Technology Innovation Platform Support Project of Zhongnan Hospital, Wuhan University (PTXM2020008), Science and Technology Innovation Cultivation Fund of Zhongnan Hospital, Wuhan University (cxpy2017043). Medical Science Advancement Program (Basic Medical Sciences) of Wuhan University (TFJC2018004).

\section{Authors' contributions}


WT and KL conceived and designed this investigation. FM and XZ helped to design the scheme of the investigation. FM and MW collected the original data. MW and SW analyzed the data. MW, WT, and KL contributed to the interpretation of the data. MW, SW, MG, WT, and KL contributed to the writing of the paper. All authors read and approved the final manuscript.

\section{Acknowledgements}

Not applicable.

\section{References}

1. Johns Hopkins Coronavirus Resource Center. https://coronavirus.jhu.edu/map.html. Accessed 21 May 2021.

2. Härter G, Spinner CD, Roider J, Bickel M, Krznaric I, Grunwald S, Schabaz F, Gillor D, Postel N, Mueller MC et al. COVID-19 in people living with human immunodeficiency virus: a case series of 33 patients. Infection 2020, 48(5):681-686.

3. Huang J, Xie N, Hu X, Yan H, Ding J, Liu P, Ma H, Ruan L, Li G, He N et al. Epidemiological, virological and serological features of COVID-19 cases in people living with HIV in Wuhan City: A populationbased cohort study. Clinical infectious diseases: an official publication of the Infectious Diseases Society of America 2020. DOI: 10.1093/cid/ciaa1186.

4. Eckerle I, Meyer B. SARS-CoV-2 seroprevalence in COVID-19 hotspots. Lancet (London, England) 2020, 396(10250):514-515.

5. Wu Z, McGoogan JM. Characteristics of and Important Lessons From the Coronavirus Disease 2019 (COVID-19) Outbreak in China: Summary of a Report of 72314 Cases From the Chinese Center for Disease Control and Prevention. JAMA 2020, 323(13):1239-1242.

6. Guo W, Ming F, Dong Y, Zhang Q, Zhang X, Mo P, Feng Y, Liang K. A Survey for COVID-19 Among HIV/AIDS Patients in Two Districts of Wuhan, China. SSRN Electronic Journal. DOI: $10.2139 /$ ssrn.3550029.

7. Guo W, Ming F, Feng Y, Zhang Q, Mo P, Liu L, Gao M, Tang W, Liang K. Patterns of HIV and SARS-CoV2 co-infection in Wuhan, China. Journal of the International AIDS Society 2020, 23(7):e25568.

8. COVID-19 Diagnosis and Treatment Guideline in China (8th ed.). http://www.gov.cn/zhengce/zhengceku/2020-08/19/content_5535757.htm. Accessed 21 May 2021.

9. Guidelines for the Prevention and Treatment of Opportunistic Infections in Adults and Adolescents with HIV. https://hivinfo.nih.gov/sites/default/files/ebook.pdf. Accessed 21 May 2021.

10. The National COVID-19 seroepidemiological Survey by Chinese center for disease control and prevention. http://www.chinacdc.cn/jkzt/crb/zl/szkb_11803/jszl_2275/202012/t20201228_223494.html. Accessed 21 May 2021. 
11. Brown LB, Spinelli MA, Gandhi M. The interplay between HIV and COVID-19: summary of the data and responses to date. Current opinion in HIV and AIDS 2021, 16(1):63-73.

12. Pan Y, Li X, Yang G, Fan J, Tang Y, Hong X, Guo S, Li J, Yao D, Cheng Z et al. Seroprevalence of SARSCoV-2 immunoglobulin antibodies in Wuhan, China: part of the city-wide massive testing campaign. Clinical microbiology and infection: the official publication of the European Society of Clinical Microbiology and Infectious Diseases 2020. DOI: 10.1016/j.cmi.2020.09.044.

13. Health Commission of Hubei Province. Novel Coronavirus Epidemic Prevention and Control 97th Press conference.

http://wjw.hubei.gov.cn/bmdt/ztzl/fkxxgzbdgrfyyq/xxfb/202005/t20200519_2277791.shtml. Accessed 21 May 2021.

14. Long Q-X, Tang X-J, Shi Q-L, Li Q, Deng H-J, Yuan J, Hu J-L, Xu W, Zhang Y, Lv F-J et al. Clinical and immunological assessment of asymptomatic SARS-CoV-2 infections. Nat Med 2020, 26(8):12001204.

15. Menghua W, Xin Z, Jianwei L, Yu Z, Qinwei Y. Case report: one case of coronavirus disease 2019 (COVID-19) in a patient co-infected by HIV with a normal CD4 T cell count. AIDS Res Ther 2020, $17(1): 46$.

16. Suwanwongse K, Shabarek N. Clinical features and outcome of HIV/SARS-CoV-2 coinfected patients in The Bronx, New York city. Journal of medical virology 2020, 92(11):2387-2389.

17. Vizcarra P, Pérez-Elías MJ, Quereda C, Moreno A, Vivancos MJ, Dronda F, Casado JL. Description of COVID-19 in HIV-infected individuals: a single-centre, prospective cohort. The lancet HIV 2020, 7(8):e554-e564.

18. Shalev N, Scherer M, LaSota ED, Antoniou P, Yin MT, Zucker J, Sobieszczyk ME. Clinical Characteristics and Outcomes in People Living With Human Immunodeficiency Virus Hospitalized for Coronavirus Disease 2019. Clinical infectious diseases: an official publication of the Infectious Diseases Society of America 2020, 71(16):2294-2297.

19. Mascolo S, Romanelli A, Carleo MA, Esposito V. Could HIV infection alter the clinical course of SARSCoV-2 infection? When less is better. Journal of medical virology 2020, 92(10):1777-1778.

20. Pallikkuth S, de Armas L, Rinaldi S, Pahwa S. T Follicular Helper Cells and B Cell Dysfunction in Aging and HIV-1 Infection. Front Immunol 2017, 8:1380.

21. Xiao Y, Wu S, Marly G, Ming F, Wu M, Tang W, Liang K. People Living with HIV Easily Lose Their Immune Response to SARS-CoV-2: Result from a Cohort of COVID-19 Cases in Wuhan, China. DOI: 10.2139/ssrn.3774187.

22. Ghosn J, Taiwo B, Seedat S, Autran B, Katlama C. HIV. Lancet (London, England) 2018, 392(10148):685-697.

23. Can Sarınoğlu R, Sili U, Eryuksel E, Olgun Yildizeli S, Cimsit C, Karahasan Yagci A. Tuberculosis and COVID-19: An overlapping situation during pandemic. Journal of infection in developing countries 2020, 14(7):721-725. 
24. Adepoju P. Tuberculosis and HIV responses threatened by COVID-19. The lancet HIV 2020, 7(5):e319e320.

25. Marimuthu J, Kumar BS, Gandhi P A. HIV and SARS CoV-2 coinfection: A retrospective, record-based, case series from South India. Journal of medical virology 2020. DOI: 10.1002/jmv.26271.

26. B-Lajoie M-R, Drouin O, Bartlett G, Nguyen Q, Low A, Gavriilidis G, Easterbrook P, Muhe L. Incidence and Prevalence of Opportunistic and Other Infections and the Impact of Antiretroviral Therapy Among HIV-infected Children in Low- and Middle-income Countries: A Systematic Review and Metaanalysis. Clinical infectious diseases: an official publication of the Infectious Diseases Society of America 2016, 62(12):1586-1594.

27. Beck BR, Shin B, Choi Y, Park S, Kang K. Predicting commercially available antiviral drugs that may act on the novel coronavirus (SARS-CoV-2) through a drug-target interaction deep learning model. Computational and structural biotechnology journal 2020, 18:784-790.

28. Gao K, Nguyen DD, Wang R, Wei G-W. Machine intelligence design of 2019-nCoV drugs. bioRxiv: the preprint server for biology 2020. DOI: 10.1101/2020.01.30.927889.

29. Inciarte A, Gonzalez-Cordon A, Rojas J, Torres B, de Lazzari E, de la Mora L, Martinez-Rebollar M, Laguno M, Callau P, Gonzalez-Navarro A et al. Clinical characteristics, risk factors, and incidence of symptomatic coronavirus disease 2019 in a large cohort of adults living with HIV: a single-center, prospective observational study. AIDS (London, England) 2020, 34(12):1775-1780.

30. Cao B, Wang Y, Wen D, Liu W, Wang J, Fan G, Ruan L, Song B, Cai Y, Wei M et al. A Trial of LopinavirRitonavir in Adults Hospitalized with Severe Covid-19. The New England journal of medicine 2020, 382(19):1787-1799. 